\title{
STABLE HOMOTOPY THEORY IS NOT SELF-DUAL ${ }^{1}$
}

\author{
J. M. BOARDMAN
}

\begin{abstract}
The classical Spanier-Whitehead duality for finite complexes shows that the finite stable homotopy category is selfdual. We prove that in the larger stable categories, duality is not consistent with the standard arguments of homotopy theory.
\end{abstract}

One motivation for introducing stable homotopy theory is Spanier-Whitehead duality [2]. This duality implies that a certain stable category formed from finite cell complexes is self-dual. For other purposes, however, this stable category has to be enlarged in some way. We prove that Spanier-Whitehead duality does not hold in the larger category of spectra $S_{h}$ introduced in [1]. Our proof shows more, that this failure is inherent in any stable theory rich enough to allow the usual procedures of homotopy theory.

We refer to [1] freely. Finite spectra $X$ enjoy the property $[1, \mathrm{C} 26]$ that $\left\{X, \vee_{i} Y_{i}\right\}=\oplus_{i}\left\{X, Y_{i}\right\}$ for any family of spectra $Y_{i}$, in other words (see $[1, \mathrm{C} 20])$ that any morphism from $X$ to an infinite sum factors through some finite subsum. Let us call a spectrum $X$ with the dual property "cofinite", that is, $\left\{\prod_{i} Y_{i}, X\right\}=\bigoplus_{i}\left\{Y_{i}, X\right\}$ for any family of spectra $Y_{i}$ (products exist by $[1, \mathrm{C} 22]$ ). We find there are not enough cofinite spectra for duality.

TheOREM. The category $S_{h}$ is not equivalent to its dual category. In fact the only cofinite spectra are contractible.

Proof. Suppose $X$ is a noncontractible cofinite spectrum.

(1) By $[1, \mathrm{~L} 5], X$ has a nonzero homotopy group.

(2) We take $Y_{i}=S^{i}$, the $i$-sphere, for $i>0$. Then the wedge $\bigvee_{i} S^{i}$ also serves as the product $\prod_{i} S^{i}$ in $S_{h}$ by $[1, L 7]$, so that $\left\{\prod_{i} S^{i}, X\right\}$ $=\prod_{i}\left\{S^{i}, X\right\}$. It follows that $\pi_{i}(X)=0$ for all sufficiently large $i$.

(3) Let $G=\pi_{n}(X)$ be the top nonzero homotopy group of $X$, which exists by (1) and (2). By obstruction theory, $\{Y, X\} \cong \operatorname{Hom}\left(\pi_{n}(Y), G\right)$ whenever $Y$ is $(n-1)$-connected. Since $\pi_{n}(Y)$ is arbitrary and we can choose each $Y_{i}$ to be $(n-1)$-connected, we find

Received by the editors January $21,1970$.

AMS 1969 subject classifications. Primary 5525, 5540.

Key words and phrases. Stable homotopy, Spanier-Whitehead duality, finite spectrum, infinite sum, infinite product.

1 Research partially supported by the Army Research Office (Durham) grant DA-31-124-ARO(D)-176. 


$$
\operatorname{Hom}\left(\prod_{i} H_{i}, G\right)=\underset{i}{\bigoplus} \operatorname{Hom}\left(H_{i}, G\right)
$$

for any family of abelian groups $H_{i}$. This imposes strong conditions on $G$.

(4) $G$ does not contain the cyclic group $\boldsymbol{Z}_{p}$ as a subgroup, for any prime $p$. Take $H_{i}=Z_{p}$ for all $i$, so that $\oplus_{i} H_{i} \subset \prod_{i} H_{i}$ are vector spaces over the field $\boldsymbol{Z}_{p}$. Then any homomorphism $\oplus_{i} H_{i} \rightarrow G$ extends over $\prod_{i} H_{i}$, which contradicts (3) unless $\operatorname{Hom}\left(\boldsymbol{Z}_{p}, G\right)=0$. Hence $G$ is torsion-free.

(5) $G$ does not contain the group $\boldsymbol{Q}$ of rationals, by replacing $\boldsymbol{Z}_{p}$ by $Q$ throughout (4).

(6) If $A \rightarrow B \rightarrow C \rightarrow A$ is an exact triangle and $A$ and $B$ are cofinite, then by the five-lemma $C$ is also cofinite. We take $A=B=X$ and $\gamma: A \rightarrow B$ as $p$ times the identity class, which we may do by $[1, \mathrm{~J} 11]$. (We construct $C$ as the mapping cone of $\gamma$.) Then by exactness, since $\gamma_{*}: \pi_{*}(X) \rightarrow \pi_{*}(X)$ is also multiplication by $p, \pi_{*}(C)$ consists only of $p$-torsion, which contradicts (4) for $C$ unless $\pi_{*}(C)=0$. Thus $\gamma_{*}$ must be an isomorphism, and the group $G$ must be divisible.

From (4), (5) and (6) we have a contradiction on $G$, since any torsion-free divisible group is a rational vector space. Thus $G$ and $X$ do not exist.

\section{REFERENCES}

1. J. M. Boardman, Stable homotopy theory, University of Warwick, November 1965 (mimeograph).

2. E. H. Spanier, Duality and S-theory, Bull. Amer. Math. Soc. 62 (1956), 194-203. MR 19, 51.

The Johns Hopkins University, BaLtimore, Maryland 21218 